\title{
Speeding Up Navigational Requests in a Parallel Object Database System
}

\author{
Jim Smith ${ }^{1}$, Paul Watson ${ }^{1}$, \\ Sandra de F. Mendes Sampaio ${ }^{2}$, and Norman W. Paton ${ }^{2}$ \\ 1 Department of Computing Science, University of Newcastle upon Tyne, \\ Newcastle upon Tyne, NE1 7RU UK \\ \{Jim.Smith, Paul.Watson\}@ncl.ac.uk \\ 2 Department of Computer Science, University of Manchester, \\ Manchester, M13 9PL UK \\ \{sampaios, norm\}@cs.man.ac.uk
}

\begin{abstract}
In data intensive applications, both programming and declarative query languages have attractions, the former in comprehensiveness and the latter for ease of use. Databases sometimes support the calling of side-effect free user defined functions from within declarative queries. As well as enabling more efficient coding of computationally intensive functions, this provision not only moves computation to data in a clientserver setting, but also enables speedup through data parallel execution if the server is parallel. There has been little work on the combined use of query and program based database access in the context of parallel servers. We believe Polar is the first parallel object-oriented database which supports this arbitrary navigation both in a client application and in functions (operations) which may be called from within declarative queries. This work introduces Polar's support for navigation and for calling operations from within parallel queries and presents performance results for example navigational requests for the large OO7 benchmark.
\end{abstract}

\section{Introduction}

Suppose a large engineering design company has a collection of engine designs to be stored in a database, and that an engine is modelled as a hierarchical collection of component objects, such as a gearbox, cylinders etc, each having $3 \mathrm{~d}$ shape and orientation. Suppose also a client seeks a particular type of engine that fits into some space which has arbitrary $3 \mathrm{~d}$ shape, and can tolerate various possible orientations. The overall request is suited to expression in a declarative query language, but the computation which determines whether a particular engine fits into the available space may be too complex, or too CPU intensive, to express in a high level query language. On the other hand the request could be implemented entirely in a programming language. In this case however, not only must the implementor handle the data related optimisation issues which the query compiler would handle for a declarative expression, but in the typical client-server context the cost of transporting the data comprising all the engine 
designs to the client is incurred. If the server is parallel, then there is a greater incentive to execute complex code on the server since the query language part of the request is transparently executed in parallel, offering the potential to gain a speedup in execution of the complex/CPU intensive portion of the request expressed in a programming language, even though that language is sequential, by calling the code on multiple objects in parallel.

The need to combine query and programming languages to meet user requests is widely appreciated, and stored procedures in SQL [9] support the calling of programming language code from within an SQL query. In 12 a four quadrant classification is presented of database applications, with those in the fourth quadrant, that require both query and programatic access, being considered the most challenging. The engine design example postulated above is typical of those problems in this class where, not only the query language part of the request, but also the programming language part needs to access complex stored data. The Object Database Management Group (ODMG) standard [6] defines a marriage of Object Query Language (OQL) and programming languages (e.g. $\mathrm{C}++$ ) for an OODB that addresses these problems and others in the fourth quadrant. In an OODB, the complex computation of the example described earlier could be expressed as an operation of the Engine class, such as Engine::fitsInto(...), implemented in a programming language, such as $\mathrm{C}++$, and called both from a client based navigational program and from an OQL expression.

There is not much work on parallel database support for both navigational and programatic access. Monet is a parallel OODB, optimised for main memory use, but while supporting a library of parallel traversals [1], it doesn't provide for arbitrary navigation in a programming language. Parsets [13] are sets of object identifiers (OIDs) which support global operations on the referenced objects which are distributed over multiple Shore [5] instances. When such an operation is called, the OIDs are distributed to their home processors and the operation performed there. One of these operations, Apply allows a method of each object to be called. While Parsets implement certain global operations familiar in query languages, such as Select and Reduce, execution is planned explicitly by the user rather than by a query compiler based on a full physical algebra and exploiting data related statistics maintained in the database metadata.

Saving previous results e.g. [8,10], can benefit performance of queries calling user-defined functions where a particular function is called with the same parameters multiple times, but not where a particular function is called for the same object multiple times with different parameters, or where multiple functions are called for the same object. The work described here is concerned with situations where functions must be called and so employs the complementary approach of object state caching.

Polar [1], unlike Monet is disk based and supports programming language bindings in the ODMG style, and unlike the Parsets approach integrates operation calls into a physical algebra supporting OQL. Polar claims to be the first parallel disk based ODMG compliant architecture implemented. While earlier work on Polar [1134] emphasised query processing facilities, this paper expands 
the architectural description to show how navigation is supported and presents measurements for a prototype implementation.

In the following, Section 2 gives an overview of the Polar architecture, while Section 3 describes its support for OQL based operation calls. By way of example, Section 4 describes how selected navigational requests may be parallelised, and Section 5 presents measured results for these examples implemented on a prototype of the Polar architecture. Finally, Section 6 concludes.

\section{Architecture}

As illustrated in 11, the Polar parallel store comprises multiple store units accessing global metadata, but distributed application data, each comprising an object manager and query execution engine. The object store may be accessed by both an OQL client and a navigational client, the latter executing an application written in a programming language. Either may generate OQL query expressions, which it directs to a compiler unit.

Logically, data is organised by extents which are directly accessible to code, be it a declarative query or a navigational program, executed on either the server or a client machine. Physically however, these extents are partitioned across a number of volumes distributed over a number of store units. Documenting this partitioning in the metadata allows both the query compiler and a navigational operator, using the volume mapping information also stored in the metadata, to identify the store units containing partitions of a particular extent.

An OQL expression undergoes logical, physical and parallel optimisation to yield a data flow graph of parallel algebra operators (query plan), which is distributed between object store and client. Typically one partition of this plan runs in the client and the remainder in a selection of store units, the latter creating and filtering tuples, former collating and presenting tuples. A tuple is a complex but generic structure which holds an instance of an intermediate result collection within an executing query plan.

The operators follow the iterator model [7] whereby each implements a common interface comprising three main functions: open performs setup and causes the operator to begin computing its result collection; next returns the next tuple in that result; and close is called to clean up, after receipt of an eof tuple. As described in 11, parallelism in a query is encapsulated in the exchange operator which implements a partition between two threads of execution, and a configurable data redistribution, the latter implementing a flow control policy.

Figure 1 shows the runtime support in a navigational client and store unit. Both contain a query execution engine, support for language binding and query setup/control together with some basic infrastructure. Control is initiated in the client based application, but following query compilation the resulting query plan is controlled by execution services in the separate machines while the application waits for results to stream through.

In the store unit, programming language code is encapsulated within operation libraries that are loaded on demand under control of such an executing 


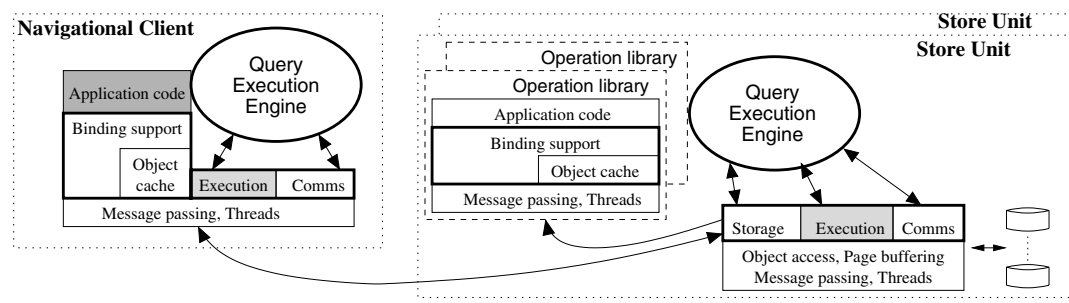

Fig. 1. Runtime Support Services

query. The language binding support, which contains an object cache which transparently faults in objects as required, is also present in a query compiler and an OQL client to support access to the distributed metadata. In general, the execution of declarative queries is planned to access data once only so there is little scope for exploiting an object cache. As described elsewhere [4], where objects are reaccessed many times in a query, the approach adopted in Polar is to access data at a lower level than the object cache in scans and to use specific tuple caching where this is beneficial.

The query execution engine implements the algorithms of the parallel algebra operators, building on a support library. At the lowest level is basic support, for: management of untyped objects, message exchange and multi-threading. On top of this, a storage service supports page based access to objects either by OID directly or through an iterator interface to an extent partition and a communications service implements the flow control underlying exchange. In support of inter store navigation, the storage service can request and relay pages of objects stored at other store units.

\section{Operation Calls}

Schema compilation generates a description of the application schema, including operations within the metadata. From this, a header file in the appropriate programming language can be derived to be included when compiling operation implementations and application code. Each operation body conforms to its definition in the header file. An operation is called from within a query via a stub file which converts between data representations used within the execution engine and those used within language bindings and constructs the call parameters. Specifically, a different representation of complex structures and collections is used in the query execution engine from those of the language bindings. A stub generator automatically creates this code from the metadata. Currently in Polar, an operation is globally identified by a signature comprised of its fully scoped name and parameter types, for example:

\section{OO7::Document::replaceText(IN(string),IN(string))}

The signature is stored in the metadata entry for the corresponding operation. 


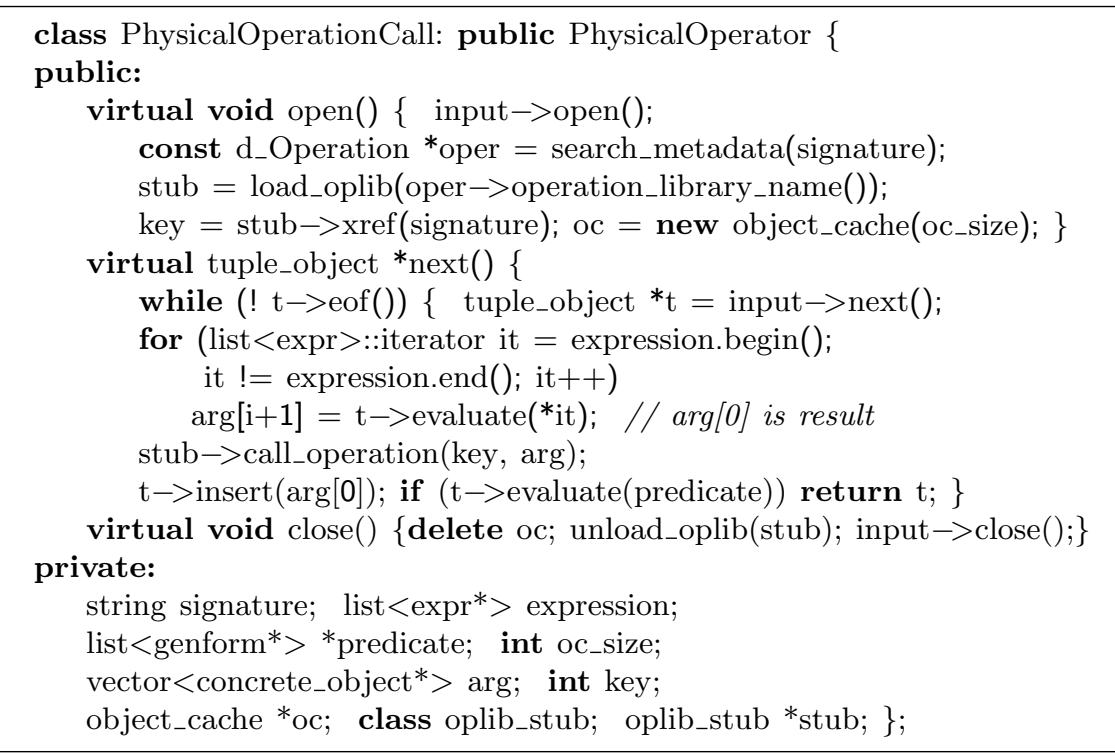

Fig. 2. Implementation of a simple Operation call operator

Compiled operation implementations can be linked into either a client program or, with an appropriate stub, a shared library. The installation utility records the name of the operation library in the metadata describing each of the operations implemented in that library.

Execution of such operation calls in OQL is realised through a separate physical operator, operation call. This facilitates arbitrary placement of a call within the query plan. Figure 2 outlines a realisation of a simple operation call operator. An object cache is created in open(), shared by all operation calls made in next(), and finally shut down in close(). The appropriate size for a particular object cache may be determined by user hint and/or runtime statistics. The stub code is responsible for actually initiating an operation call, by first creating a reference of the correct type in the object cache from the OID stored in the tuple, and then calling the appropriate method through that reference.

The basic parameters of the simple Operation call operator are:

- the signature of the function to be executed;

- the name by which the result is to be known in the result tuple;

- a list of (possibly constant) expressions, identifying actual parameters;

- optionally, a predicate to filter tuples resulting from the operation call; and

- parameters defining the environment in which the function call is executed, for instance the size of the object cache. 


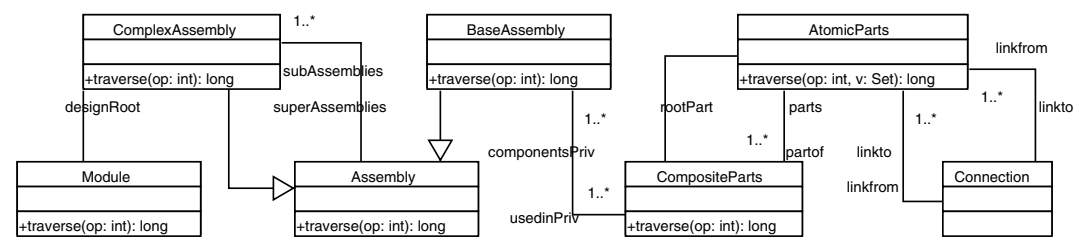

Fig. 3. A possible representation of $t 1$ and $t 6$ in the database schema

\section{Parallelising Navigational Requests}

To illustrate the parallelisation of navigational requests, the following shows how two navigational requests of the OO7 benchmark [2] may be executed first sequentially and then in parallel. The examples chosen are traversals $t 1$ and $t 6$ which perform read only traversals of the design hierarchy, in the former case completing a depth first traversal of the atomic parts in each composite part and in the latter case touching only the single root atomic part of each composite part. Figure 3 shows how the two traversals might be reflected in a single operation traverse $(o p)$ at each level in the design hierarchy, where $o p$ is an integer value selecting traversal 1 or 6 . Figure 4 shows $\mathrm{C}++-\mathrm{OML}$ code implementing these operations.

Originally OO7 [2] specified the traversal benchmarks with respect to a single module. As in the Parsets work, where a configuration having multiple modules is used, traversals are applied to all those modules. However, no ordering of the sep-

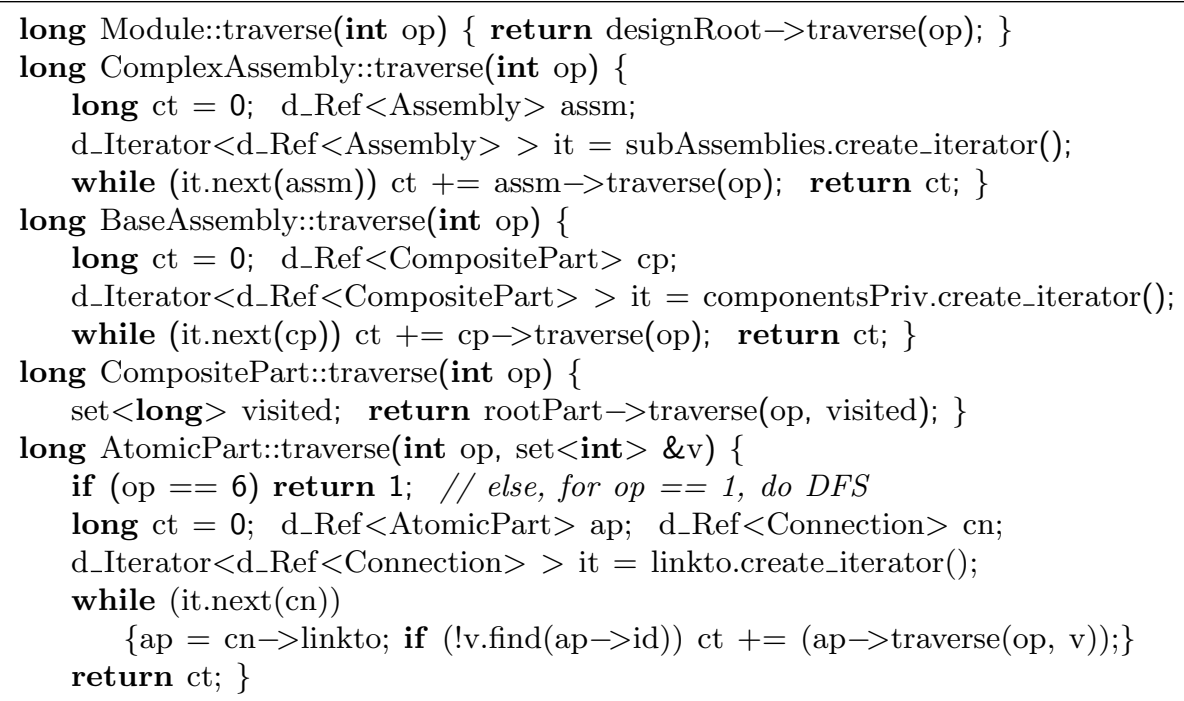

Fig. 4. Implementation of $t 1$ and $t 6$ in $\mathrm{C}++-\mathrm{OML}$ 


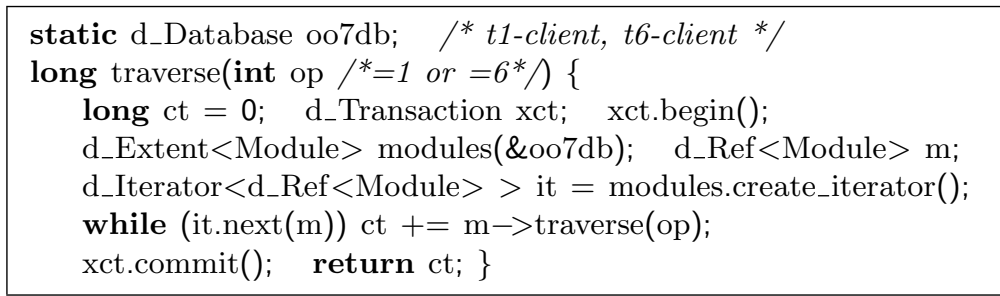

Fig. 5. $\mathrm{C}+-\mathrm{OML}$ client code to call $t 1$ or $t 6$

arate module traversals is specified, though each module traversal is assumed to be depth first. In the original benchmark presentation a navigational client program iterates through the extent of modules and calls the Module::traverse(...) operation of each. Figure 5 shows essential parts of such client $\mathrm{C}++-\mathrm{OML}$ code.

OQL does not currently support a general form of recursion such as that required to traverse the assembly hierarchy purely within OQL. However, for a given database size it is possible to unroll the recursion by hand. In the main three OO7 configurations where there are 7 levels of assembly objects $t 6$ may be implemented using the OQL expression below.

select count(c.rootPart.id) /*t6-q2*/

from $\mathrm{m}$ in modules, a 2 in m.designRoot.subAssemblies, a3 in a2.subAssemblies, a4 in a3.subAssemblies, a5 in a4.subAssemblies, a6 in a5.subAssemblies, a7 in a6.subAssemblies, $\mathrm{b}$ in a7.subAssemblies, $\mathrm{c}$ in b.componentsPriv;

Alternatively, either request may be implemented by appropriately calling Module::traverse(...) within an OQL query.

\begin{tabular}{|c|c|}
$\begin{array}{c}\text { select m.traverse(1) } / * t 1-q 1 * / \\
\text { from } m \text { in modules; }\end{array}$ & $\begin{array}{c}\text { select } m \text {.traverse(6) } / * t 6-q 1 * / \\
\text { from } m \text { in modules; }\end{array}$ \\
\hline
\end{tabular}

A possible parallel query plan corresponding to the OQL expression $t 1-q 1$ is shown in figure 6. The query is distributed across the parallel store by executing sub-plan 1 in each store processor and sub-plan 2 in the coordinator. The operation call operator receives a stream of Module OIDs and, for each of these, calls

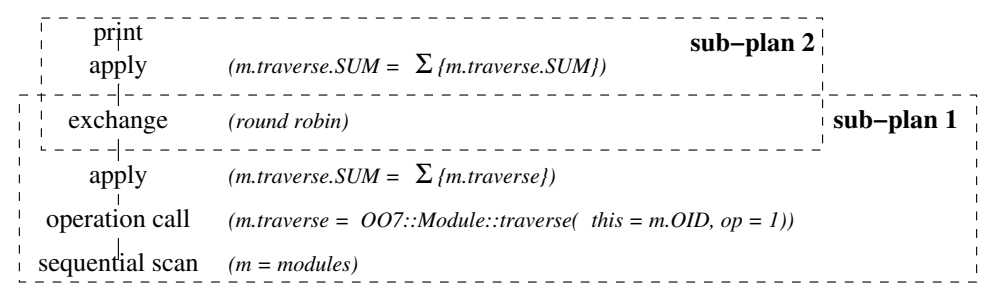

Fig. 6. A possible parallel query plan implementing the OQL expression $t 1-q 1$ 
the operation $007::$ Module::traverse(1). The call returns are summed locally and these local sums are accumulated in the coordinator.

\section{Experimental Evaluation}

Initial results are reported here for the implementations of the traversals described in section 4. The experiments are conducted using instantiations of the OO7 database on Polar within a shared nothing environment comprising a cluster of $10860 \mathrm{MHz}$ Pentium III machines running RedHat Linux version 7.2, each with $512 \mathrm{MB}$ main memory and local disks, connected via a $100 \mathrm{Mbps}$ Fast ethernet hub. For each experiment, data is partitioned over a number of machines but located on one disk per machine, this being a SEAGATE ST39204LC. User requests are submitted, except where otherwise indicated, from an identical but separate machine on the same network. The OO7 configuration used is large with fan-out 3 and occupies a total of about 750M Bytes.

Polar's bulk loading facility supports a number of data distribution policies including the ability to cluster by store unit one object with a related object. In these experiments, modules are distributed round robin across the store units and all remaining levels of the OO7 hierarchy, i.e. assemblies, manuals, composite parts, etc, are clustered with the related module. This clustering policy ensures that all inter store traffic is confined to the physical algebra operators, so it is easier to understand the expected performance. However, since there are only 10 modules in large OO7, the performance graphs show obvious granularity effects.

Figure 7 (a) compares the measured performance of the three implementations of $t 6: t 6$-client in the single store configuration; $t 6-q 1$ and $t 6-q^{2}$ across the range of parallel configurations. The performance of $t 6-q 1$ is better than
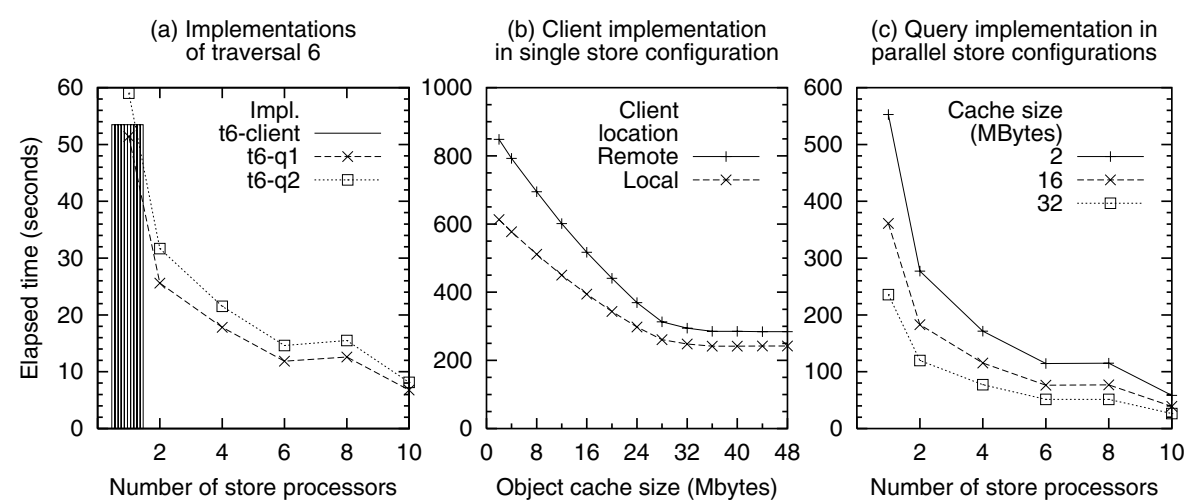

Fig. 7. Measured performance over large OO7 (a) of implementations of $t 6$ (b) of t1-client, running in separate cases remotely from the store unit and locally, and (c) of t1-q1. In (a), the client performance, shown as a bar, is only measured over the single store configuration 
that of $t 6$-client even in the single store configuration. This is to be expected since the operation code in $t 6$-client is running remotely and therefore pays some cost for data transfer across the network while in $t 6$ - $q 1$, the same operation code is running in the same context as the server process.

Logically the two parallel implementations are performing identical traversals, but the implementations are quite different. The following are likely to contribute to the higher cost of the OQL based implementation.

- The object store is built on Shore [5] and the disk format of object states is closer to that of the $\mathrm{C}++$ binding than to that of the generic tuple structure, so the cost of object materialisation is likely to be higher in the latter case.

- The version of Polar used for these experiments did not include the tuple caching facility of 4. Therefore, while $t 6$ - $q 1$ can re-access cheaply from the object cache composite part and atomic part objects which are touched more than once in the traversal, $t 6-q 2$ must repeat the tuple materialisation multiple times, even if all such objects are retained in file-system buffers or in the storage manager buffer pool

Overall in this experiment highest performance is achieved through encoding the bulk of the user level query in class operations, installed on the server and called via an OQL query. It seems likely that this pattern will recur in other examples, but set against the performance advantage of the installed operations is the administrative overhead and development cost.

Figure 7 (b) shows how the performance of the sequential implementation, t1-client, varies with object cache size for the single store onfiguration where the client is located: firstly on a separate machine from the store; and secondly on the same machine as the store. Figure 7 (c) shows the performance of the parallel implementation, $t 1-q 1$, on different store configurations.

Assuming that traversing an already cached composite part has zero cost, an upper bound on the reduction in the cost of the overall traversal as the cache size is increased from 'small' to 'large' may be obtained by considering the degree of reuse of composite parts in base assemblies. In large OO7, there are 21870 references to 5000 composite parts, so the value of this upper bound is 4.4 . The reductions seen in Figures 7 (b) and (c) are less than this bound, but $2 \mathrm{MB}$ is the smallest size setting currently supported in the object cache, and the cost of accessing an object from cache will not strictly be zero.

In the single store configuration of Figure 7 (c), some improvement over the performance of the local client in Figure [7 (b) is evident, reflecting the optimisation of RPCs to local procedure calls in the former. The speedup over the remote client, single store, execution, for similar object cache size, is over 10 for the parallel implementation when the database is distributed over 10 store processors. In fact, the speedup is over 9 with respect to the local client for each of the object cache sizes. 


\section{Conclusions}

This paper has described the implementation and evaluation of a mixed query and programming language environment over a parallel OODB. The work has shown how the query and procedural languages present in a parallel OODB can be combined to speedup operations which traverse complex structures of objects. Furthermore, experimental results have shown good absolute speedup in benchmark tests. We believe that Polar is the first implementation of a parallel OODB which supports this combination. In these experiments, a highly favourable data distribution is employed. Furthermore, the load is as balanced as possible, allbe-it at the large granularity of a module. Further work will explore these issues, along with that of implementing more complex user requests, e.g. ones implying multiple operation calls.

\section{References}

1. P. A. Boncz, F. Kwakkel, and M. L. Kersten. High performance support for OO traversals in Monet. In BNCOD, pages 152-169. Springer, July 1996.

2. M. J. Carey, D. J. DeWitt, and J. F. Naughton. The OO7 benchmark. In SIGMOD Conference, pages 12-21. ACM Press, May 1993.

3. S. de F. Mendes Sampaio, J. Smith, N. W. Paton, and P. Watson. An experimental performance evaluation of join algorithms for parallel object databases. In EuroPar. Springer, August 2001.

4. S. de F. Mendes Sampaio, J. Smith, N. W. Paton, and P. Watson. Experimenting with object navigation in parallel object databases. In Workshop on Parallel and Distributed Databases, with DEXA'01, Munich, Germany, August 2001.

5. M. J. Carey et al. Shoring up persistent applications. In SIGMOD Conference, pages 383-394. ACM Press, 1994.

6. R. G. G. Cattell et al., editor. The Object Database Standard: ODMG 2.0. Morgan Kaufmann, San Francisco, 1997.

7. G. Graefe. Query evaluation techniques for large databases. ACM Computing Surveys, 25(2):73-170, June 1993.

8. A. Kemper, C. Kilger, and G. Moerkotte. Function materialization in object bases: Design, realization, and evaluation. TKDE, 6(4):587-608, August 1994.

9. J. Melton. Understanding SQL's Persistent Stored Modules. Morgan Kaufmann, San Francisco, 1997.

10. J. M. Hellerstein J. F. Naughton. Query execution techniques for caching expensive methods. In SIGMOD Conference, pages 423-434. ACM Press, June 1996.

11. J. Smith, P. Watson, S. de F. Mendes Sampaio, and N. W. Paton. Polar: An architecture for a parallel ODMG compliant object database. In $C I K M$, pages 352-359. ACM Press, November 2000.

12. M. Stonebraker and P. Brown. Object Relational DBMSs Tracking The Next Great Wave. Morgan kauffman, September 1998.

13. D. J. De Witt, J. F. Naughton, J. C. Shafer, and S. Venkataraman. Parallelising OODBMS traversals: A performance evaluation. VLDB Journal, 5(1):3-18, January 1996. 